\title{
Development of Conceptual Design for Electric Bus Body Structure Using Simple Structural Surface Method
}

\author{
Ismoyo Haryanto ${ }^{1,2^{*}}$, Achmad Widodo ${ }^{1}$, Ibrahim Satya ${ }^{1}$, Gunawan Dwi Haryadi ${ }^{1,2}$, Ojo Kurdi ${ }^{1,2}$, \\ Bentang Arief Budiman',3, Sigit Puji Santosa ${ }^{2,3}$ \\ ${ }^{1}$ Department of Mechanical Engineering, Faculty of Engineering, University of Diponegoro, Semarang, Indonesia \\ ${ }^{2}$ National Center for Sustainable Transportation Technology, Indonesia \\ ${ }^{3}$ Faculty of Mechanical and Aerospace Engineering, Institut Teknologi Bandung, Indonesia \\ *Email: ismoyoharyanto@lecturer.undip.ac.id
}

\begin{abstract}
An optimum design for a vehicle structure is always desired because the structure can significantly affect the vehicle's performance. However, some complex iterations are usually involved in the designing process. The objective of the present study is to implement the Simple Structural Surfaces (SSS) method for analyzing electric bus body structure that can reduce complexity in the stage of conceptual design. The SSS method model the vehicle structure as several planar sheets and determine the forces in each sheet. Implementing the SSS method at the early stage of the vehicle's development can minimize the number of parameter changes needed during the late stage of development. The results showed that compared with the results obtained from FEM, the SSS method gave the maximum stress value on the chassis in good accordance. Yet, the downside of using this method is that determining the deflections in the structure becomes a little bit complicated. Successfully implementing this strategy can reduce the time and cost required to develop an effective vehicle structure.
\end{abstract}

\section{Keywords}

Bus body; Conceptual design; Structure; Simple structural surface; Electric bus

\section{Introduction}

It was reported that several countries have targeted to achieve net-zero emissions by 2050. Based on a study of the International Energy Agency (IEA), it would mean that total $\mathrm{CO}_{2}$ emissions have to be reduced by around $45 \%$ from 2010 levels by 2030 . Therefore, a large number of changes across the energy sector would need to be realized simultaneously, at a time when the world is recovering from the pandemic of Covid-19. IEA has also examined several individual measures related to behavior, which in total would reduce $\mathrm{CO}_{2}$ emissions. The majority of these reductions are in the transport sector, reducing $\mathrm{CO}_{2}$ emissions by more than $20 \%$. [1]

Although, without replacing fossil-fuel power plants, transport electrification does not lead to decreasing emissions that contribute to climate change mitigation, implementing electrified road transport with appropriate actions in other sectors of the economy permitted an optimistic outlook for a low-carbon transition. Some studies showed that the mitigation cost generated by the $2{ }^{\circ} \mathrm{C}$ climate stabilization target could be reduced by penetration of electric vehicles so that the climate change mitigation does not have to occur at the expense of economic growth. Therefore, electrification is widely considered an attractive solution for reducing the oil dependency and emissions of road transportation
[2-4]. In densely populated areas, electric vehicles are a key technology to reduce air pollution and a promising option to contribute to energy diversification and greenhouse gas emissions reduction objectives [5].

With Covid-19, urban public transport, including buses, in addition to facing challenges in providing highcapacity and affordable services, must also ensure health security. In densely populated cities of developing and developed countries, where heavy congestion occurs, urban buses provide an irreplaceable means of transportation. Hence, the balancing between the pandemic impacts, the overall capacity of the urban transport system, and sustained government support are necessary for the future of public transit in general and electric buses in particular [5].

To achieve the two-degree Celsius scenario (2DS) goal, in which the average global temperature increases by 2050 is limited to only $2^{\circ} \mathrm{C}$, a proper engineering analysis is mandatory in the design and manufacturing of electric vehicles. However, there is a drawback in electric vehicle development, i.e., the battery life between charges is still the biggest problem. To resolve that issue, the lightweight design of the electric vehicle is a compromise method because it will increase energy-consumption efficiency and decrease the required battery capacity. Therefore, light and compact 
structural designs are highly significant for electric vehicles [6-10].

A structural design in automotive manufacturing is a crucial factor in the performance of the vehicle. The structure must carry the applied loads that consist of all vehicle subcomponents [11-13]. However, the structural design of a vehicle is very complex. There are lots of changes required even in the latest development phase of the design. Traditionally at the stage of the design phase, methods of trial and error are widely applied. On the other hand, trial and error methods will be a very risky approach since, at the stage of the design phase, the required changes to the structure are dependent on the vehicle components. These changes can be very expensive and cost a lot of time and resources when a comprehensive analysis process during the conceptual design stage is implemented. This is why it will be profitable to implement simple analyses at the early design phase.

There are many simplified methods for body modeling in the conceptual design stage. Wood, Barari, and Esmailzadeh, in 2014 have used the Simple Structural Beam (SSB) Model to optimize and analyze automotive structures for bending stiffness and natural frequency [14]. In this method, the vehicle is modeled as several beam elements. A finite element method is used to solve the deflections, reaction forces, and internal loading on each element of the structure. This Simple Structural Beam model can be easily adapted to allow optimization of the static property of the structure bending stiffness. Dynamic properties of the vehicle structure are also examined through vibration analysis by determining the fundamental natural frequency of the structure. By implementing these methods, the structural stiffness can be increased in bending compared to the original design [14]. Meanwhile, Tebby, Barari, and Esmailzadeh have developed a method that uses beam elements to represent the vehicle structure. This method was used to optimize the design based on the torsion stiffness of the structure. The results showed that more than $60 \%$ increase of the structural torsion stiffness to weight ratio when comparing an initial design to a design with optimum selection of the design parameter. This method can be efficiently employed for the initial design of vehicle structure when weight reduction and enhance of structural stiffness are the major objectives [15].

A simple structural surfaces (SSS) model for vehicle model test-bending has been developed by Mohd Nor et. al. The model has been implemented for a sedan model. The result shows that the application of this simplified approach is highly beneficial in the development of modern passenger car structure design.
It is clearly observed that the global bending stiffness reduces significantly when more panels are removed from the complete SSS model. The analysis shows the front parcel shelf is an important subassembly to sustain bending load [16].

A modeling method of the body using the simple structure surface was also proposed by Xiu-Hua Zhang et. al. In that study the structure surface model of the half-integral electric car body was built as well as by using transfer path analysis the structure surfaces are balanced in the bearing force, force transfer is consecutive, and the structure surface model is integrated. In addition, the force matrix equations in the bending and torsional conditions were derived, which are the basis of computing the simplified structure surface model [17].

In the present research, the SSS model for analyzing electric bus body structure will be developed. This model is expected to be able to be applied at the conceptual design stage of the electric bus. In this study, modeling of forces occurring in the upper structure, as well as analysis of force due to bending on the idealized structure, was carried out. It was assumed that the structural load is to be evenly distributed over all parts of the bus, all loads are static conditions and the reviewed electric bus is medium size.

\section{Simple Structural Surface Method}

The simple structural surface (SSS) method was introduced by Dr. Janusz Pawlowski and applied to the design of passenger vehicles, buses, and trams. The SSS method is a method used at the stage of conceptual design or when there are fundamental changes to the structure. The principle is that the vehicle structure being reviewed is modeled as a number of surface planes. Although modern passenger vehicles require attractive aerodynamics and models to have a surface with high curvature, they can still be approached with components or subassemblies represented as surface planes.

The SSS is a structural element plane or subassembly that can be considered rigid in its plane. The forces at each surface area must be in balance. The force can be generated from the components that are on it, such as the rail which is connected to a structural member, which produces a paired force to maintain the balance of the force. The procedure for determining the load on the SSS is to be passed through the structure from one axle to another until the overall equilibrium of the structure is reached. So this method is very useful for determining that there is continuity of the direction of forces and determining the integrity of the structure. 
Figure 1 shows an example of the SSS method surface plane. Like many other models, the structural model built with SSS will not fully understand the structural behavior. However, this method makes it possible to know the type of loading conditions applied to each member of the vehicle's main structure. The drawback of this model is that it cannot be applied to the redundant structure as well as for statically indeterminate structures.

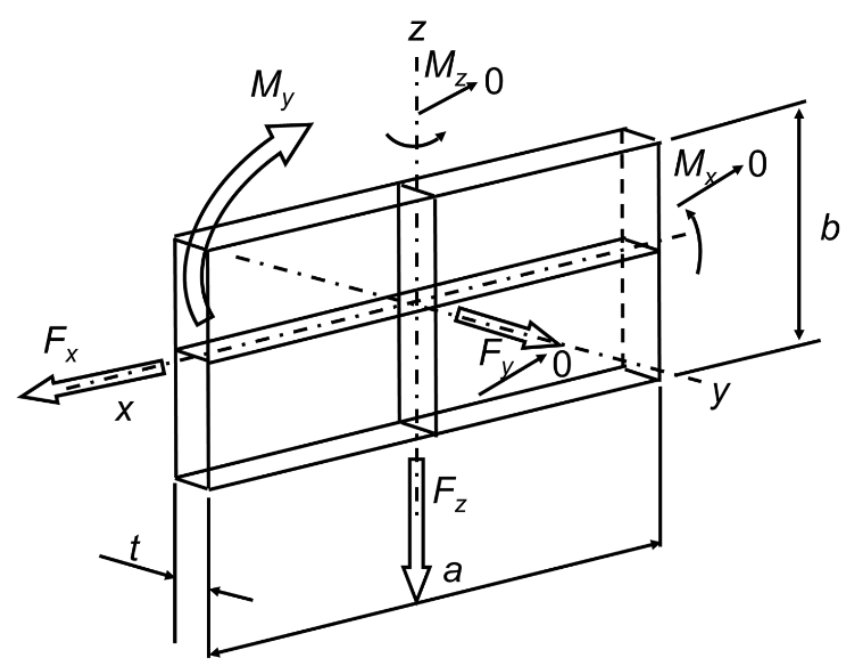

Figure 1 Definition of a simple structure surface [11]

\section{Development of SSS Model for Electric Bus Body Structure}

In the present study, the SSS model will be implemented on a medium electric bus body structure. The design of SSS structures in this work must be able to define the deflection of the SSS model subjected to bending loading, calculate the overall bending stiffness of the vehicle, and provide an overview of the structural integrity of the vehicle. A complete design of the SSS model is divided into two parts of the upper body and the lower part bearing the upper body. The upper body is used to bear passengers and cargo. Meanwhile, the lower part is used to bear the battery, motor, and its controller. The stiffness of the upper body structure is relatively lower than the stiffness of the bottom bearing body. The higher bending and torsion stiffness and larger torsion strength of the bottom bearing body are needed to ensure that the foundation bearing body is small in bending and torsion deformation, thus the battery will not be extruded due to the structural deformation of the body. The connection between the upper and lower bodies is rigid.

During the early design stage, it is recommended to start first with the bending and torsion load cases with SSSs at the total body structure level instead of the body subunit level. The suggested steps are as follow [11]:
1) Estimate the loads and loading conditions.

2) Draw free body and loading diagrams.

3) Calculate the internal, non-edge reaction forces.

4) Construct shear and bending moment diagrams.

5) Formulate the matrix equations to solve for the edge forces.

Steps 1 to 4 should be repeated for local subunits and components. For the first stage of the present study only bending load will be considered.

\section{Case Study}

The electric bus considered herein is designed for urban transport with a capacity of 28 seated passengers. The bus is $2.55 \mathrm{~m}$ wide, $8 \mathrm{~m}$ long, and $3.2 \mathrm{~m}$ high. Figure 2 shows an outside perspective of the bus body model. As shown in the figure, the bus separated into the top part and the bottom part. The top part also includes the cover panel of an air conditioner, while the bottom part is connected to the front and rear axles. The openings are designed for the passenger door, emergency exit, and windows. The bus interior comprises passenger seats that are anchored to the floor panel. Battery packs are stored in the compartment beneath the floor. The total mass of an 8-m bus excluding the weight of the rear axle, front axle, and tires, is about 5.8 tons. The total mass of passengers is estimated as 1.82 ton which is distributed in seven rows along the bus. The battery placement is divided into front and rear compartments with each of $200 \mathrm{~kg}$ of estimated mass. Table 1 shows a breakdown of the masses of the electric-bus components.

Table 1 Mass breakdown of medium bus components

\begin{tabular}{lr}
\hline \multicolumn{1}{c}{ Item } & Mass (kg) \\
\hline Right Frame & 241.76 \\
Left Frame & 241.76 \\
Roof Frame & 169.16 \\
Floor Frame & 179.86 \\
Driver \& Co & 130 \\
Passenger & 1820 \\
Motor & 340 \\
Battery A & 200 \\
Battery B & 200 \\
Air-conditioning system & 175 \\
Chassis $\quad 2120$ \\
\hline \multicolumn{2}{c}{ Total } \\
\hline
\end{tabular}

Figure 3 shows masses of the major components, passengers, luggage, and distributed body mass of the electric bus being studied. The longitudinal positions of these masses are shown relative to the vehicle body. First, calculate the front and rear axle reactions. Using the diagram as depicted by Figure 3 combined with the equations of equilibrium the front and rear axle reactions can be obtained. For the model of the electric bus body structure studied in this paper, the front and 
rear axle reactions were $\mathrm{F}_{\mathrm{F}}=17.342 \mathrm{kN}$ and $\mathrm{F}_{\mathrm{R}}=40.833$ $\mathrm{kN}$, respectively. The center of gravity of the bus body structure can also be calculated and found $4.247 \mathrm{~m}$ behind the bumper.

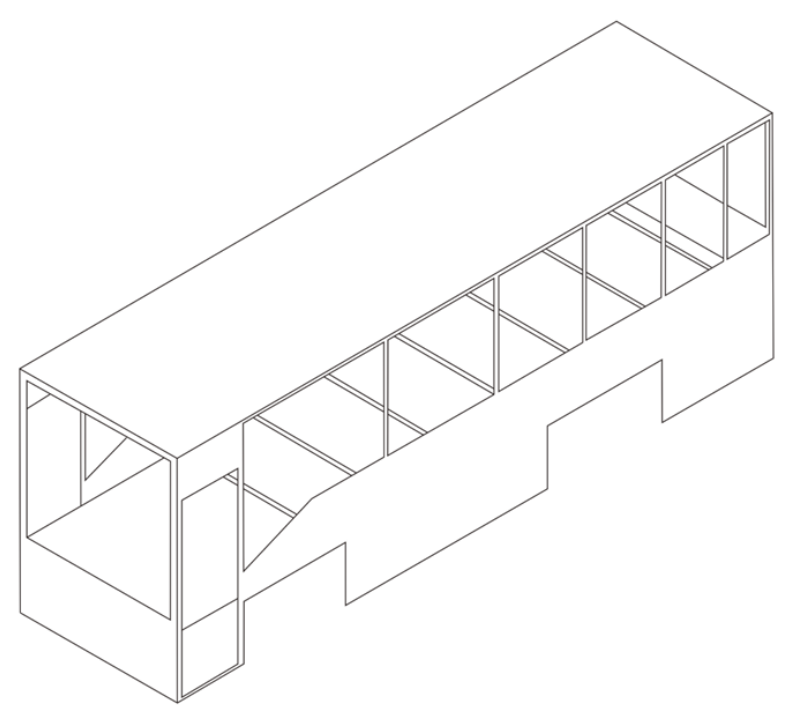

Figure 2 The outside perspective of the bus body model

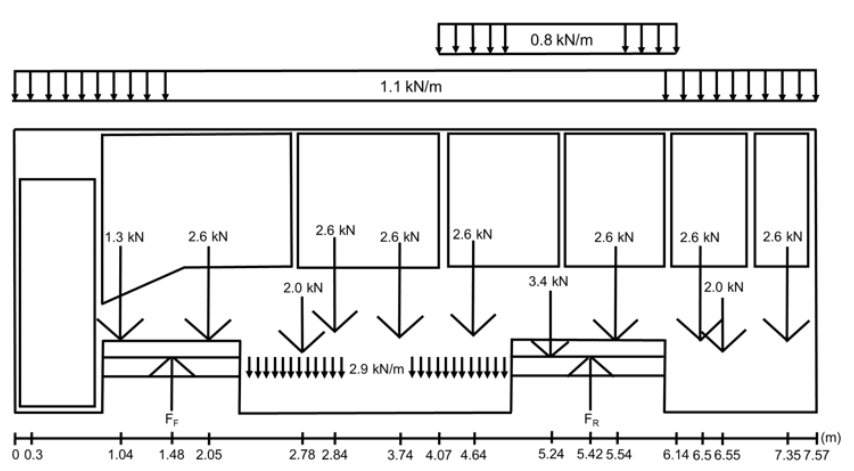

Figure 3 Free body diagram of medium bus body structure

Finally, it must be decided where the loads must be applied to the various SSSs. In the present study, only the bending test will be considered. This test is performed by applying a force to each chassis connection to the frame. This simulates when the bus passes a speed bump, creating a bending force on the entire bus structure. Furthermore, the forces are evaluated on each surface that composes the model. By resolving the force on each surface, the shear force and bending moment diagram can be constructed. Figure 4 shows the load conditions for the SSS model of the electric bus body structure in the present study.

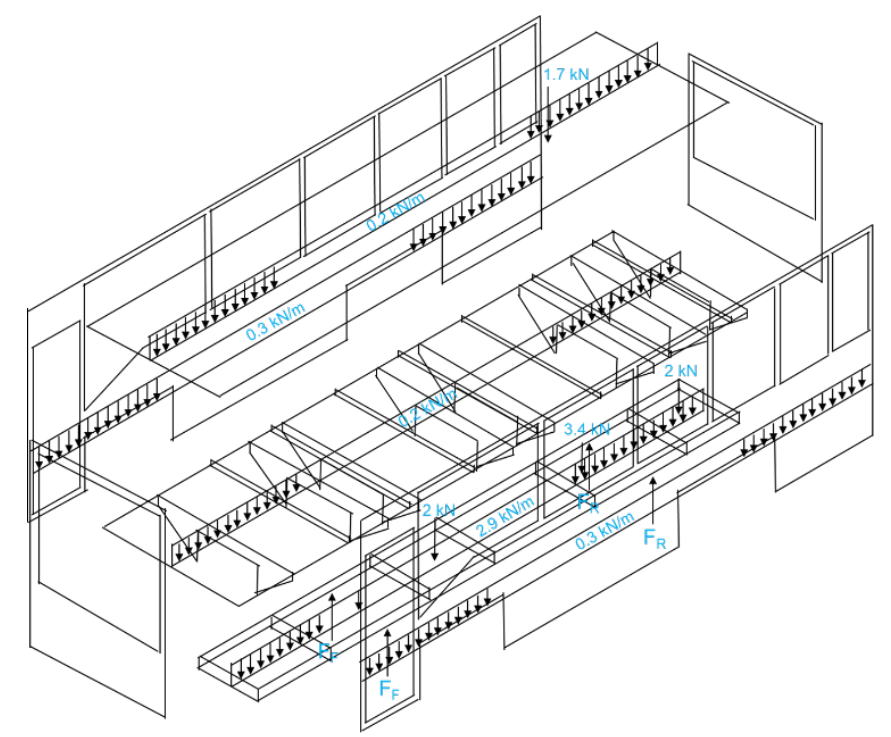

Figure 4 Bending load for SSS model

A complete picture of the overall loading condition of the structure has now been determined so the next stage is to determine the loads on each SSS. Force calculation begins from the chassis section. Chassis is part of the bus that supports the overall structure. In this section, there are chassis connections to the upper structure, motor, battery, and axle. The joints on the chassis with the upper structure consist of eight joints. Figure 5 shows the force distribution on the chassis. It can be seen that the force distribution on the front axle is $29.8 \%$ and on the rear axle is $70.2 \%$. Meanwhile, the diagrams of shear and bending moment are depicted in Figure 6. It can be seen in Figure 6 that the maximum shear and bending moment are $66.76 \mathrm{kN}$ at $\mathrm{x}=5.88 \mathrm{~m}$ and $29.04 \mathrm{kN} \mathrm{m}$ at $\mathrm{x}=6.69 \mathrm{~m}$, respectively. In this paper, only the seventh chassis joint to the upper structure is considered. Figure 7 shows the force distribution of the seventh chassis joint and its shear and bending moment distributions are shown by Figure 8 . As shown by Figure 8 that the maximum value for the shear of the seventh connection joint is $112.31 \mathrm{kN}$ and for the bending moment is $65.38 \mathrm{kN} \mathrm{m}$.

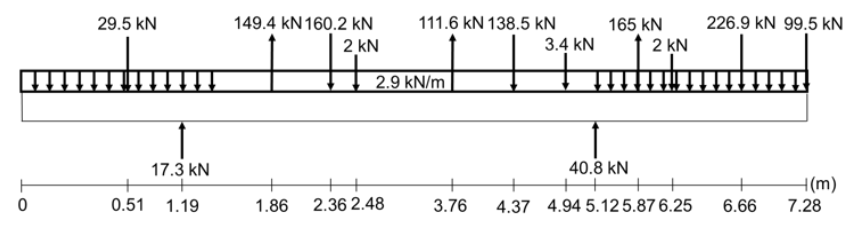

Figure 5 Chassis load distribution 

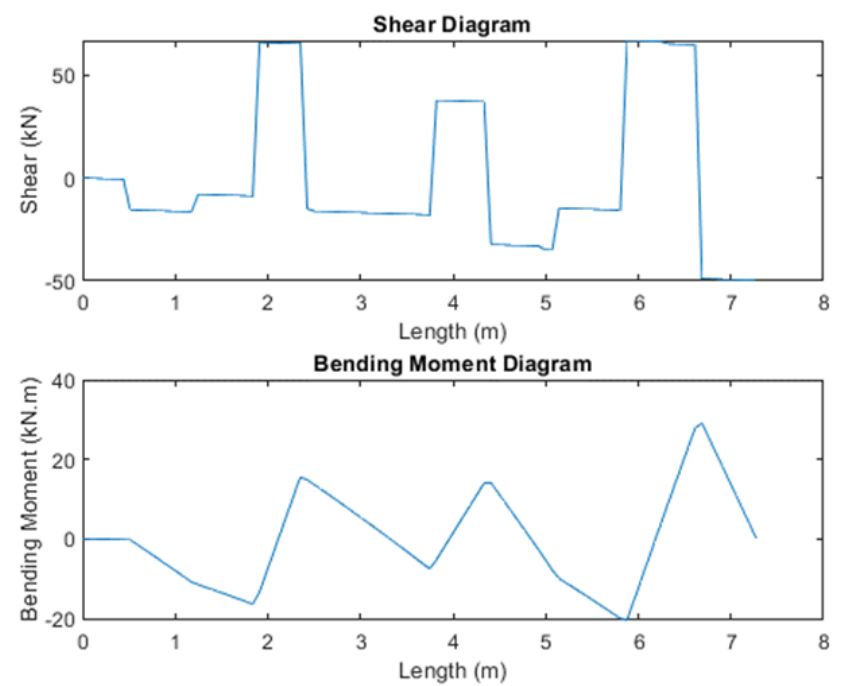

Figure 6 Shear and bending moment distribution on chassis

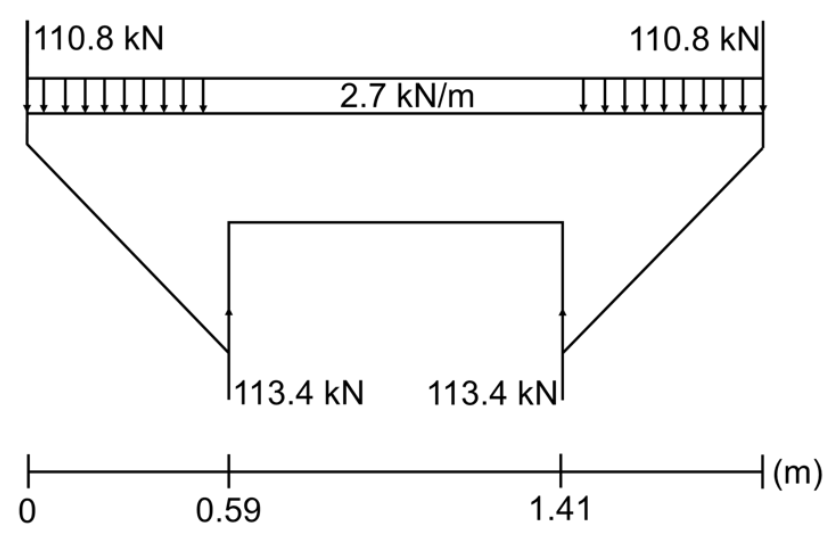

Figure 7 The seventh connection load
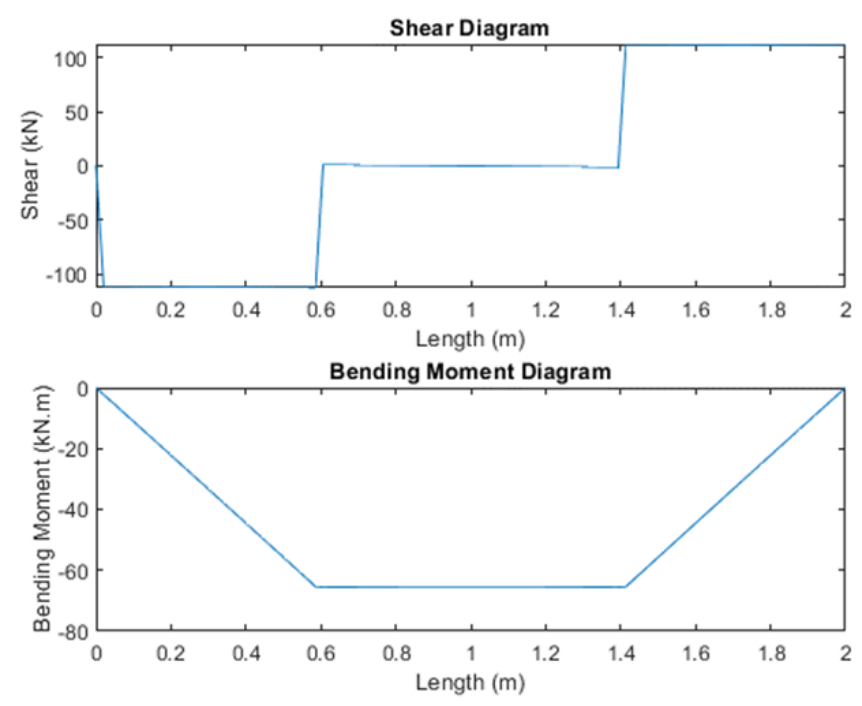

Figure 8 Shear and bending moment distribution on the seventh connection load
From all the previous analyses the loads that have been determined are transferred out to the side frame from the various SSSs. In addition, the side frame is assumed to carry the distributed loads of the body mass plus some additional loads applied directly from the passengers/seats. In Figure 9 we have the values of all the forces acting on the side frame. Finally, the shear force and bending moment diagrams are plotted in Figure 10. Figure 10 show that the maximum shear is $64 \mathrm{kN}$ at $x=6.89 \mathrm{~m}$ and the maximum bending moment is $27.51 \mathrm{kN} \mathrm{m}$ at $x=$ $6.963 \mathrm{~m}$.

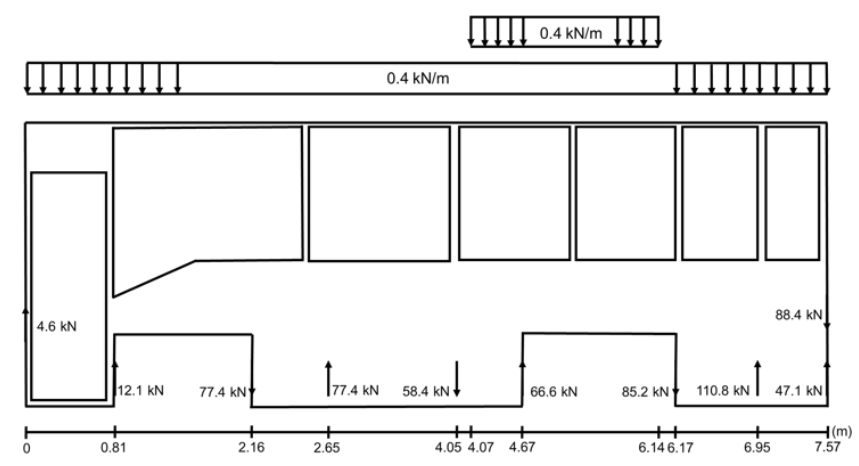

Figure 9 Side frame loads
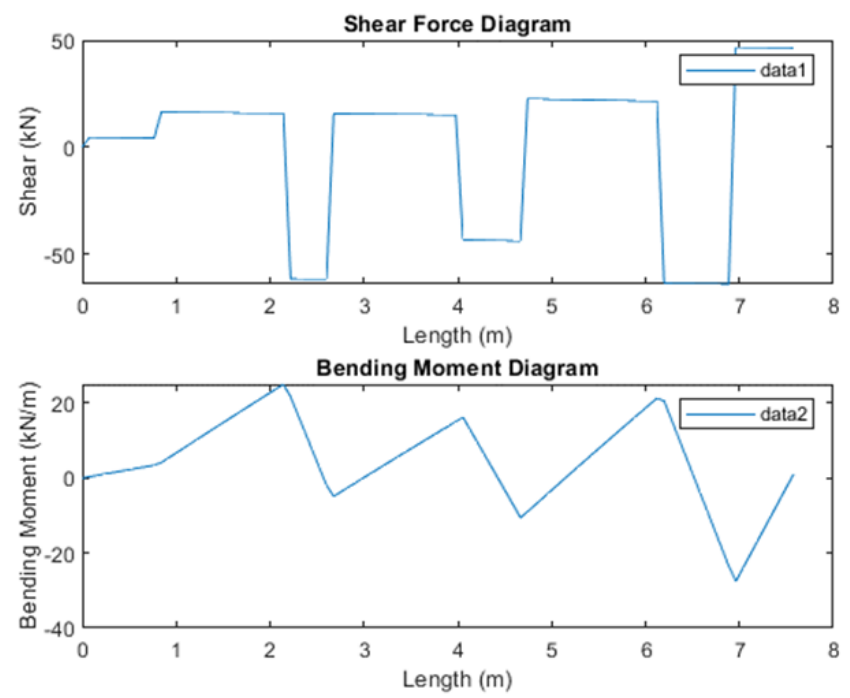

Figure 10 Shear and bending moment distribution on the side frame

\section{$5 \quad$ Validation and Discussion}

In this paper, the validation was conducted by comparing the results of the present study using FEM. For simplicity, the comparison was conducted only for the value and location of the maximum stress on the chassis. A FEM modeling was constructed, and it consists of 64505 elements. The working forces were defined at predetermined points and the constraints were located on the front and rear axle supports as shown in Figure 11. As mentioned before that, in the 
present study, only static analysis due to bending load will be considered. Figure 12 shows the stress distribution of the chassis structure obtained using FEM simulation. It can be seen that the maximum stress occurred at the rear part of the chassis with the value of $917.27 \mathrm{~N} / \mathrm{mm}^{2}$. On the other hand, the maximum stress on the chassis based on this study was calculated with reference to the bending moment distribution as depicted in Figure 6 where the maximum bending moment is $29.04 \mathrm{kN} \mathrm{m}$. It is known that the crosssection of the chassis frame has an area moment of inertia of $3.33 \times 10^{6} \mathrm{~mm}^{4}$ and a depth of $200 \mathrm{~mm}$ thus it was found that the maximum stress on the chassis frame is $876.37 \mathrm{~N} / \mathrm{mm}^{2}$. Comparing this result with the result obtained from the analysis using FEM, it appears that there is an error of $4.46 \%$.

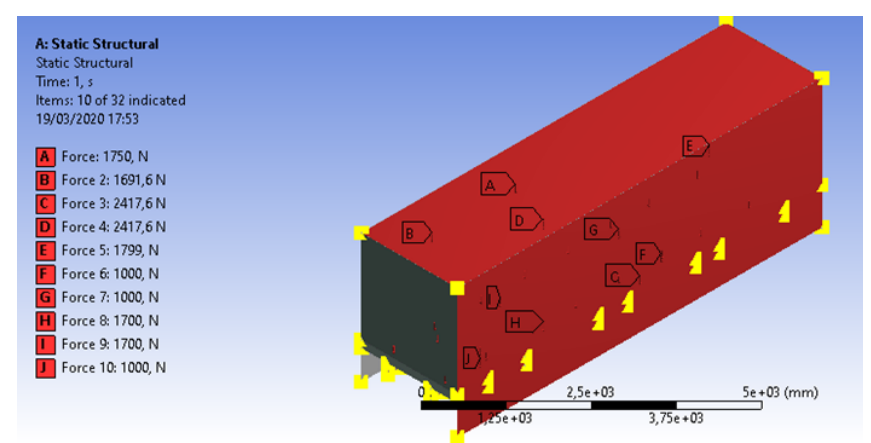

Figure 11 Determination of loads and constraints

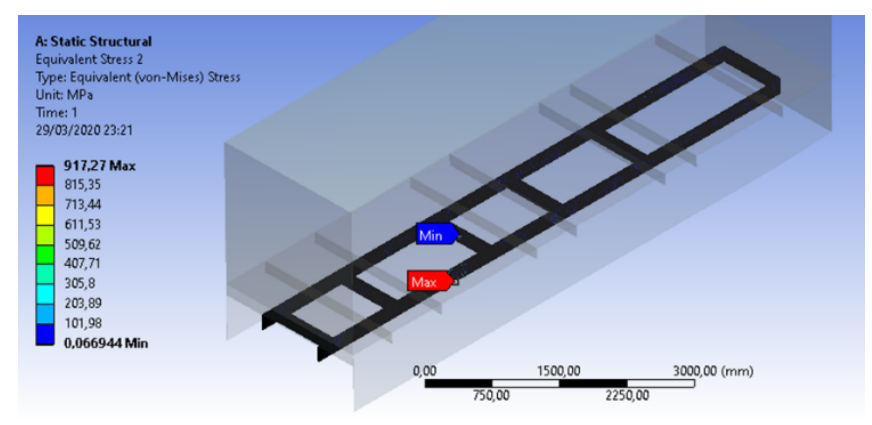

Figure 12 FEM simulation results

From the comparison, it is obvious that the SSS analysis has an almost small difference in maximum stress value to that obtained using FEM. The advantage of using the SSS model comparing to FEM for body structure analysis is that the computation time is faster. This is very beneficial, especially in the early stages of designing the vehicle body. At the beginning of the concept design phase, the available data often change quickly over a fairly short period. Many design alternatives may be under consideration. In the beginning stage of a new vehicle design, there are usually not enough data available to assemble a complete FE model of the new structure. Models that can be assembled at this early stage may be coarse. The
SSS method used prior to and during the early design stage can complement computer-aided synthesis. Therefore, the SSS method is more preferable in the first design stages.

\section{Conclusion}

This paper proposes a simple method to establish the medium electric bus body concept model based on the simple structure surface (SSS) method. The SSS method is offered to organize the process for rationalizing the basic vehicle body structure load paths. In this method, the internal force matrix equations under bending conditions are proposed by mechanical analysis and are simplified according to the simplification principle of structure surface. The internal force matrix equation will be used to obtain shear forces on each edge of surfaces. After verification, the forces on the structure surface are in balance. The results also showed that the maximum stress occurred at the rear part of the chassis and comparing to the result obtained from the analysis using FEM showed in good accordance. The results prove that the proposed vehicle model test is useful to physically demonstrate the importance of providing a continuous load path using the necessary structural components within the vehicle structures. The application of this simplified approach is highly beneficial in developing modern passenger car structure design. The downside of using this method is that determining the deflections in the structure is more complicated.

\section{Acknowledgment}

This research was funded by the Indonesia Endowment Fund for Education (LPDP) under Research and Innovation Program (RISPRO) for electric vehicle development with contract no. PRJ-85/LPDP/2020.

\section{References}

[1] International Energy Agency, "World Energy Outlook 2020," 2020. [Online]. Available: https://www.iea.org/reports/world-energy-outlook2020. [Accessed: 18-Jan-2021].

[2] R. Zhang and S. Fujimori, "The role of transport electrification in global climate change mitigation scenarios," Environ. Res. Lett., vol. 15, no. 3, p. 034019 , 2020.

[3] K. Pietrzak and O. Pietrzak, "Environmental effects of electromobility in a sustainable urban public transport," Sustainability, vol. 12, no. 3, p. 1052, 2020.

[4] S. Bellocchi, M. Manno, M. Noussan, M. G. Prina, M. Vellini, "Electrification of transport and residential heating sectors in support of renewable penetration: Scenarios for the Italian energy system," Energy, vol.196, p. 117062, 2020. 
[5] International Energy Agency, "Global EV Outlook 2020," 2020. [Online]. Available: https://www.iea.org/reports/global-ev-outlook-2020. [Accessed: 18-Jan-2021].

[6] P. Kunakorn-ong, K. Ruangjirakit, P. Jongpradist, S. Aimmanee and Y. Laoonua, "Design and optimization of electric bus monocoque structure consisting of composite materials," Proc. Inst. Mech. Eng. C. J. Mech. Eng. Sci., vol. 234, no. 20, pp. 4069-4086, 2020.

[7] A. K. Ahmed, A. M. Mohamed, M. M. Metwally, and M. E. Mokhtar, "Structural and optimization analysis of a monocoque car body," Int. J. Mod. Eng. Res., vol. 7, no. 11, pp. 28-33, Nov. 2017.

[8] I. Haryanto, F. A. Raharjo, O. Kurdi, G. D. Haryadi, S. P. Santosa, and L. Gunawan, "Optimization of Bus Body Frame Structure for Weight Minimizing with Constraint of Natural Frequency using Adaptive SingleObjective Method." Int. J. Sustain. Transp. Technol, vol. 1, no. 1, pp. 9-14, 2018.

[9] C. L. Fu, Y. C. Bai, C. Lin, and W. W. Wang, "Design optimization of a newly developed aluminum-steel multi-material electric bus body structure," Struct. Multidiscip. Optim., vol. 60, no. 5, pp. 2177-2187, 2019.

[10] F. Pan, P. Zhu, and Y. Zhang, "Metamodel-based lightweight design of B-pillar with TWB structure via support vector regression," Comput. Struct., vol. 88, no. 1-2, pp. 36-44, 2010.

[11] J. C. Brown, J. A. Robertson, and S. T. Serpento, Motor Vehicle Structures, Amsterdam: Elsevier Science \& Technology, 2001.

[12] F. Arifurrahman, B. A. Budiman, and S. P. Santosa, "Static Analysis of an Electric Three-Wheel Vehicle," in Proceedings of 5th International Conference on Electric Vehicular Technology (ICEVT), pp. 218-223, Oct. 2018.

[13] S. Rahardian, I. D. Putra, and B. A. Budiman, (2021). "On the use of steel and aluminum materials for frame structure of electric trike," Indones. J. Sci. Technol., vol. 3, no. 1, pp. 9-18, 2021.

[14] I. Wood, A. Barari, and E. Esmailzadeh, "Using the Simple Structural Beam (SSB) Model to optimize and analyze automotive structures for bending stiffness and natural frequency," in International Design Engineering Technical Conferences and Computers and Information in Engineering Conference, vol. 46346, p. V003T01A039, Aug. 2014.

[15] S. Tebby, A. Barari, and E. Esmailzadeh (2013) "Optimization of vehicle structure considering torsion stiffness using simple structural beam frame-approach," Comput. Aided Des. Appl., vol 10, no. 3, pp. 413-426, 2013.

[16] M. K. Mohd Nor, A. Noordin, M. F. S. Ruzali, M. H. Hussen, and N. M. Othman, "Development of vehicle model test-bending of a simple structural surfaces model for automotive vehicle sedan," AIP Conf. Proc., vol. 1831, no. 1, p. 020031, 2017.

[17] X. H. Zhang, F. C. Sun and C. Lin, "A modeling method of the body of conceptual design phase and the application of which in pure electric car," in Proceedings of 2017 5th International Conference on Mechanics and Mechatronics (ICMM 2017), pp. 241247, 2017. 